\title{
Control of Pushing and Pulling Motion of a Wheeled Inverted Pendulum Based on a Reduced Order Observer
}

\author{
Luis Canete ${ }^{1, a}$, Sunao Kimura ${ }^{2, b}$ and Takayuki Takahashi ${ }^{3, c}$ \\ 1,2,3960-1296 Fukushima Pref. Fukushima City Kanayagawa 1, Japan \\ aluis@rb.sss.fukushima-u.ac.jp, bsunao@rb.sss.fukushima-u.ac.jp, 'taka@rb.sss.fukushima-u.ac.jp
}

Keywords: inverted pendulum robot, disturbance observer, pushing and pulling

\begin{abstract}
In this paper the control of the I-PENTAR, a wheeled inverted pendulum type robot being developed by the authors, for pushing and pulling a cart is examined. To control the movement of the object is being pushed or pulled, information regarding several external parameters, eg. Mass of the object and friction components, must be considered. In most cases these parameters are not known before hand or may change. One method of compensating for these unknown or changing external parameters is to represent them as an equivalent reaction force from the object. Our first subject of this research is to design a disturbance observer to estimate and compensate the equivalent force. Another situation is of pushing and pulling a cart with the inverted pendulum type robot traversing an inclined plane. As an initial step to solving this problem in this paper, a force application method using whole body motion of the inverted pendulum type robot is proposed. The whole body motion means changing the balance of the robot to attain a certain desired force. During application of this force the robot must remain in its stabilized or balanced state. For an inverted pendulum type robot, this instantly poses a major problem. To solve the problem, a reduced order disturbance observer is used in this paper to estimate the force applied by the robot. On the other hand, I-PENTAR is targeted for environments where it can interact with humans and so safety is a major concern. For example, in the event that an obstacle bumps the robot as it is pushing the cart, a large and sudden force estimator based on the disturbance observer is also built into the controller. Simulation and experiments using the reduced order disturbance observer and evaluation of the whole body motion force control are presented.
\end{abstract}

\section{Introduction}

Robots aimed at aiding and interacting with humans pose two conflicting problems. These robots must be able to perform a wide range of tasks that can be generalized into lifting, pushing, or pulling of objects. The power requirement for performing these tasks varies from low to high making the use of high powered actuators logical. The use of high powered actuators becomes dangerous when used around humans. This research aims to solve the counteraction between high powered actuators and safety by implementing an Inverted Pendulum Type Assistant Robot (I-PENTAR)(Fig.1a). The Inverted Pendulum topology allows the I-PENTAR system to use its static balance as leverage thereby allowing the use of lower powered actuators at the arms. The use of the I-PENTAR system for lifting loads (Fig.1b) has been researched and has been proven to be feasible [1]. This study moves forward by focusing on a safe and efficient method of pushing and pulling loads with different masses(Fig.1c). A reduced order disturbance observer is designed for measuring the external forces applied to the system. The reduced disturbance observer is then implemented on the actual system and used as a feedforward compensator to reduce the errors when disturbance in present.

\section{System Modeling}

Two DOF Inverted Pendulum Modeling. To simplify modeling of the I-Pentar, the two degree of freedom inverted pendulum model is adopted (Fig.2) and the parameters of the inverted pendulum are shown in Table 1. The governing differential equations of the system are given by Eq. 1 and Eq. 2, $\sin \psi$ and $\cos \psi$ are represented by $S_{\psi}$ and $C_{\psi}$ respectively. 


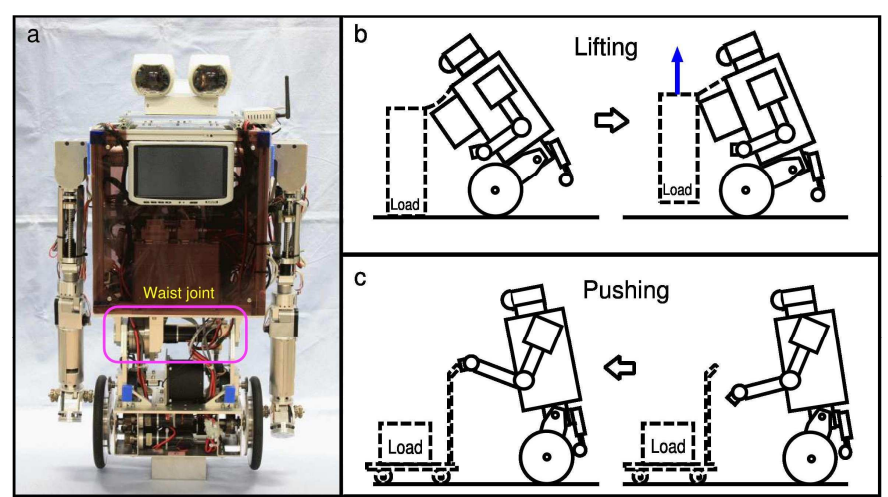

Fig. 1. Lifting and pushing and pulling tasks of I-Pentar

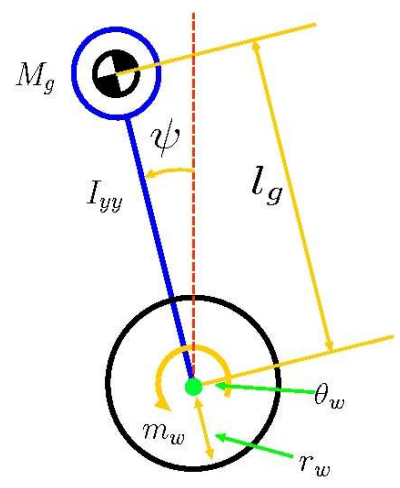

Fig. 2. Two DOF model of a wheeled inverted pendulum

Table 1 Control variables and parameters

\begin{tabular}{lll}
\hline \hline Symbol & Unit & Description \\
$\psi$ & {$[\mathrm{rad}]$} & Inclination angle of CoG \\
$\theta_{w}$ & {$[\mathrm{rad}]$} & Rotational angle of wheel \\
$M_{g}$ & {$[\mathrm{Kg}]$} & Mass of body \\
$m_{w}$ & {$[\mathrm{Kg}]$} & Mass of wheel \\
$r_{w}$ & {$[\mathrm{~m}]$} & Radius of wheel \\
& & \\
& & Length between the origin of \\
$l_{g}$ & {$[\mathrm{~m}]$} & body coordinates and CoG \\
$I_{y y}$ & {$[\mathrm{Kg}$} & $\begin{array}{l}\text { Moment of Inertia of body } \\
\text { along the Y axis }\end{array}$ \\
$I_{w a}$ & {$[\mathrm{Kg}$} & Wheel Inertia (axis) \\
& $\left.\mathrm{m}^{2}\right]$ & \\
$I_{r a}$ & {$[\mathrm{Kg}$} & Motor Inertia (axis) \\
& $\left.\mathrm{m}^{2}\right]$ & \\
& &
\end{tabular}

$$
\begin{aligned}
& \left(M_{g} l_{g}^{2}+I_{y y}+\gamma^{2} I_{r a}\right) \ddot{\psi}+\left(M_{g} r_{w} l_{g} C_{\psi}-\gamma^{2} I_{r a}\right) \ddot{\theta}_{w}+c_{w} \dot{\psi}-c_{w} \dot{\theta}_{w}-M_{g} g l_{g} S_{\psi}=-\tau_{w} . \\
& \left(M_{g} r_{w} l_{g} C_{\psi}-\gamma^{2} I_{r a}\right) \ddot{\psi}+\left\{\left(M_{g}+m_{w}\right) r_{w}^{2}+I_{w a}+\gamma^{2} I_{r a}\right\} \ddot{\theta}_{w}-c_{w} \dot{\psi}+c_{w} \dot{\theta}_{w}-M_{g} r_{w} l_{g} S_{\psi} \dot{\psi}^{2}=\tau_{w} .
\end{aligned}
$$

Modeling the disturbance. The disturbance that is applied to an inverted pendulum type robot has been modeled in previous literature [2] as a force applied on the system. The assumption that the disturbance is in the form of force is possible when the point of application of that force is constant. However, in the case of the pushing and pulling actions proposed, the torque arm of the applied force also changes (Fig. 3). This type of disturbance is instead modeled as a torque that is applied to the axle. Calculation of the applied force at the point of contact can then be performed using kinematic based solutions.

Linearization and State Space Representation and State Feedback Control. Stabilization of the inverted pendulum system was done using a linear full state feedback controller and so Eq. 1 and Eq. 2 were linearized and the disturbance torque terms were added to form Eq. 3 and Eq. 4 . Eq. 5 and Eq. 6 show the state space representation of Eq. 3 and Eq. 4.

$$
\left(M_{g} l_{g}^{2}+I_{y y}+\gamma^{2} I_{r a}\right) \ddot{\psi}+\left(M_{g} r_{w} l_{g}-\gamma^{2} I_{r a}\right) \ddot{\theta}_{w}+c_{w} \dot{\psi}-c_{w} \dot{\theta}_{w}-M_{g} g l_{g} \psi=-\tau_{w}+\tau_{d} .
$$




$$
\begin{aligned}
& \left(M_{g} r_{w} l_{g}-\gamma^{2} I_{r a}\right) \ddot{\psi}+\left\{\left(M_{g}+m_{w}\right) r_{w}^{2}+I_{w a}+\gamma^{2} I_{r a}\right\} \ddot{\theta}_{w}-c_{w} \dot{\psi}+c_{w} \dot{\theta}_{w}=\tau_{w}+\tau_{d} . \\
& \dot{\mathbf{x}}=\mathbf{A} \mathbf{x}+\mathbf{B} \tau_{w}+\mathbf{D} \tau_{d} . \\
& \mathbf{y}=\mathbf{C x} . \\
& \mathbf{A}=\left[\begin{array}{cccc}
0 & 0 & 1 & 0 \\
0 & 0 & 0 & 1 \\
a_{31} & 0 & a_{33} & a_{34} \\
0 & 0 & a_{43} & a_{44}
\end{array}\right], \mathbf{x}=\left[\begin{array}{c}
\psi \\
\theta_{w} \\
\dot{\psi} \\
\dot{\theta}_{w}
\end{array}\right], \mathbf{B}=\left[\begin{array}{c}
0 \\
0 \\
b_{3} \\
b_{4}
\end{array}\right], \mathbf{y}=\left[\begin{array}{c}
\psi \\
\theta_{w} \\
\dot{\psi} \\
\dot{\theta}_{w}
\end{array}\right], \mathbf{C}=\left[\begin{array}{llll}
1 & 0 & 0 & 0 \\
0 & 1 & 0 & 0 \\
0 & 0 & 1 & 0 \\
0 & 0 & 0 & 1
\end{array}\right], \mathbf{D}=\left[\begin{array}{c}
0 \\
0 \\
d_{1} \\
d_{2}
\end{array}\right] . \\
& \Delta=\left(M_{g} l_{g}+I_{y y}+\gamma^{2} I_{r a}\right)\left(M_{g}+m_{w}\right) r_{w}^{2}+I_{w a}+I_{r a} \gamma^{2}-\left(M_{g} r_{w} l_{g}-I_{r a} \gamma^{2}\right)^{2} \\
& a_{31}=\frac{M_{g} l_{g}}{\Delta} \quad a_{43}=\frac{c_{w}}{\Delta} \quad b_{3}=-\frac{1}{\Delta} \\
& a_{33}=\frac{c_{w}}{\Delta} \quad a_{44}=-\frac{c_{w}}{\Delta} \quad b_{4}=\frac{1}{\Delta} \\
& a_{34}=-\frac{c_{w}}{\Delta} \\
& f_{1} \psi+f_{2} \theta_{w}+f_{3} \dot{\psi}+f_{4} \dot{\theta}_{w}=\tau_{w} .
\end{aligned}
$$

The values of the disturbance matrix $\mathbf{D}$ were obtained through experimentation and the gains $\left[\begin{array}{llll}f_{1} & f_{2} & f_{3} & f_{4}\end{array}\right]$ for the state feedback controller in Eq. 7 were calculated using the Linear Quadratic Regulator (LQR).

\section{Disturbance Observer}

Disturbance as a state variable. By making the assumption that $\dot{\tau}_{d}=0$, the disturbance can be augmented into Eq. 5 and Eq. 6 so that Eq. 8 and Eq. 9 are

$$
\begin{aligned}
& {\left[\begin{array}{c}
\dot{\mathbf{x}} \\
\dot{\tau}_{d}
\end{array}\right]=\left[\begin{array}{cc}
\mathbf{A} & \mathbf{D} \\
0 & 0
\end{array}\right]\left[\begin{array}{c}
\mathbf{x} \\
\tau_{d}
\end{array}\right]+\left[\begin{array}{c}
\mathbf{B} \\
0
\end{array}\right] \tau_{w} .} \\
& \mathbf{y}=\left[\begin{array}{ll}
\mathbf{C} & 0
\end{array}\right]\left[\begin{array}{c}
\mathbf{x} \\
\tau_{d}
\end{array}\right] .
\end{aligned}
$$

Inspection of the observability matrix, derived from Eq. 8 and Eq. 9, show that is of full rank and so the system is observable.

Reduced Order Disturbance Observer. Since the system from Eq. 8 and 9 is observable, the value of $\left[\begin{array}{ll}\mathbf{x} & \tau_{d}\end{array}\right]^{T}$ can be estimated using a full order state observer. However, $\mathbf{x}$ is directly measurable so a reduced order state observer can be used to estimate the value of $\tau_{\mathrm{d}}$. Furthermore, the shape of Eq. 8 fits the shape necessary to use a reduced order disturbance observer so a transformation is not required. Derivation of the reduced order state observer is explained in [3]. Using Eq.8 and 9 the observer is implemented as

$$
\begin{aligned}
& \dot{z}=-\mathbf{L D} z+(-\mathbf{L D L}-\mathbf{L A}) \mathbf{x}-\mathbf{L B} \tau_{w} . \\
& \hat{\tau}_{d}=z+\mathbf{L x} .
\end{aligned}
$$

where $z$ is a state variable used to simplify the state equation of the observer and $\mathbf{L}$ is the observer gain. A block diagram of the disturbance observer coupled to the full state feedback controller is shown in Fig. 4. 


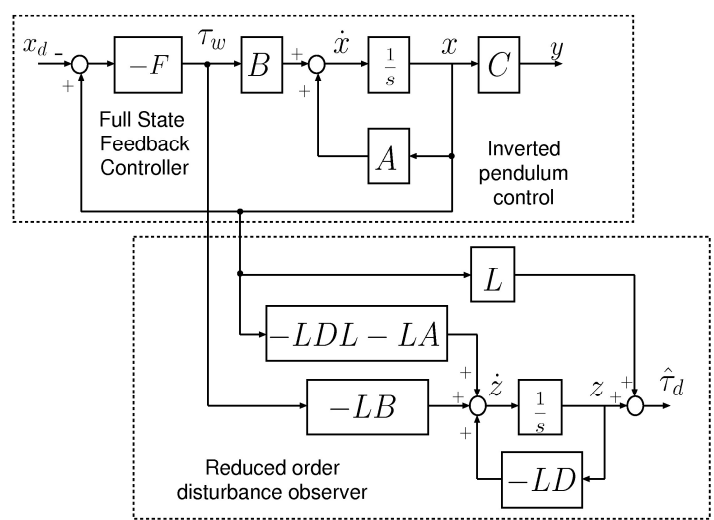

Fig. 4. Block diagram of disturbance observer coupled to full state feedback controller

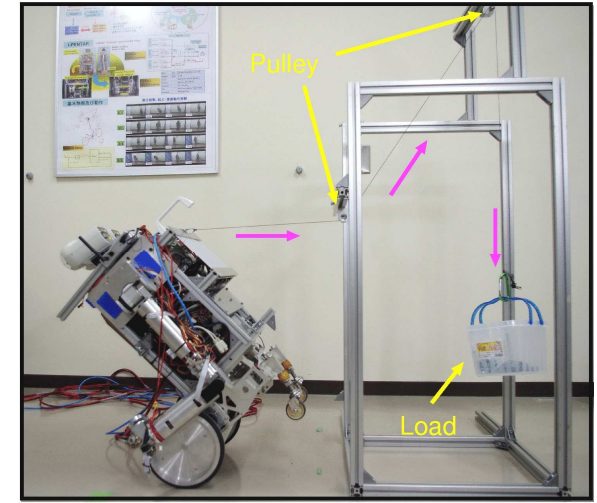

Fig. 5. Setup for testing the Disturbance Observer

Evaluation of the Disturbance Observer. To determine if the observer could estimate the disturbance, tests were conducted. The test involved applying a known force onto a predefined location on the robot making calculation of the applied disturbance torque simple. The setup of the test is shown in Fig. 5. The results show that the estimated value approaches the applied disturbance with acceptable accuracy and the settling time is approximately $4 \mathrm{~s}$ (Fig. 6).

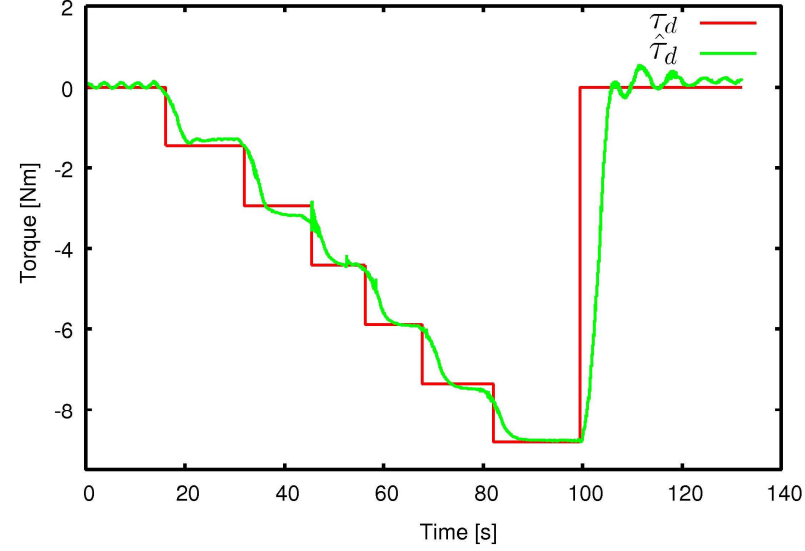

Fig. 6. Applied and estimated disturbance

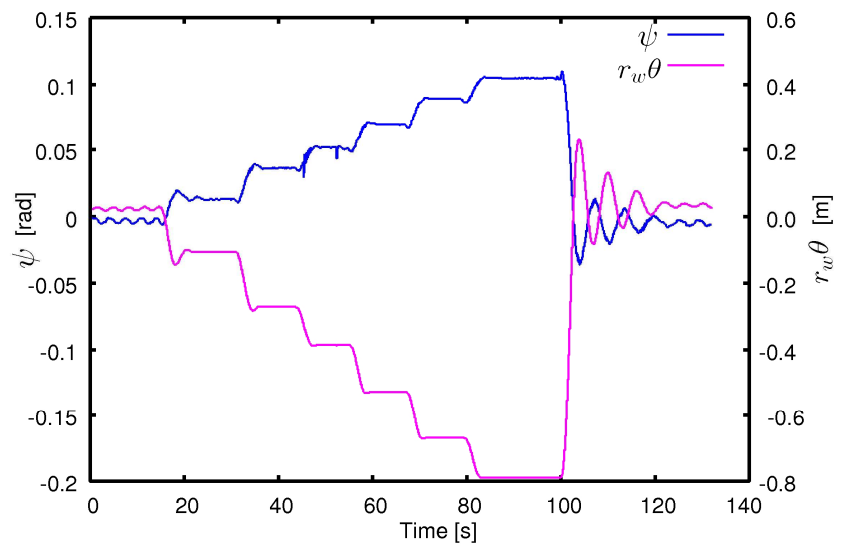

Fig. 7. Inclination angle and wheel displacement

\section{Disturbance Compensation.}

Effect of the disturbance. The effect of the disturbance can be explained by examining Eq. 3 and assuming that all acceleration and velocity terms are zero (Eq. 12). Equation 12 shows that a disturbance applied to the system causes a change in the value of the inclination angle. The state feedback controller (Eq. 7) sees the change as an error in the inclination angle and generates a torque which is opposite in direction to maintain a total moment of 0 . Assuming that the velocity terms in Eq. 7 reduce to zero, the wheel angle must become nonzero to maintain balancing. This can be seen in Fig. 7 where increased magnitudes of the disturbance also caused increase in the inclination angle and wheel displacement error.

$$
-M_{g} g l_{g} \psi=\tau_{d} \text {. }
$$

Feedforward disturbance compensation by modification of the desired wheel displacement. In [1] disturbance compensation was done by adding the estimated disturbance as a fifth term in the state feedback controller. The error in the wheel displacement is not directly caused by the disturbance but is a consequence of the state feedback controller being unable to consider the effects of the disturbance. These effects can be added into the state feedback controller by including a feedforward term $\theta_{f f}$ into the reference wheel displacement thus making the controller "aware" of the disturbance. Combining wheel displacement control and disturbance compensation into one term is convenient in 
pushing and pulling motion since the input variable in this case is displacement. Finding the expression for $\theta_{f f}$ begins by rearranging Eq. 12 to form Eq. 13. By substituting Eq. 13 into Eq. 7 and assuming that the velocity terms and total moment are 0 , the value of $\theta_{w}$ as caused by the disturbance can be found (Eq. 14). The feedforward term $\theta_{f f}$ is simply the opposite sign of Eq. 14 (Eq.15).

$$
\begin{aligned}
& \psi=-\frac{\tau_{d}}{M_{g} g l_{g}} \\
& \theta_{w}=\left(\frac{\hat{\tau}_{d} f_{1}}{M_{g} g l_{g} f_{2}}\right) \\
& \theta_{f f}=-\theta_{w}=\left(-\frac{\hat{\tau}_{d} f_{1}}{M_{g} g l_{g} f_{2}}\right)
\end{aligned}
$$

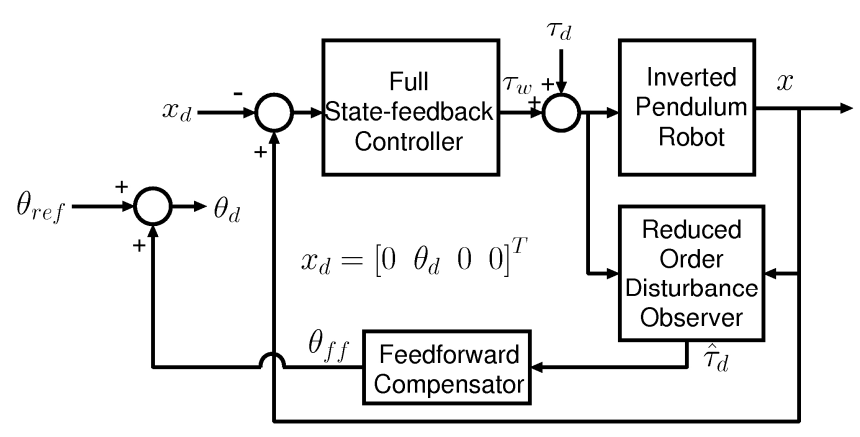

Fig. 8. Block diagram of system with feedforward compensator

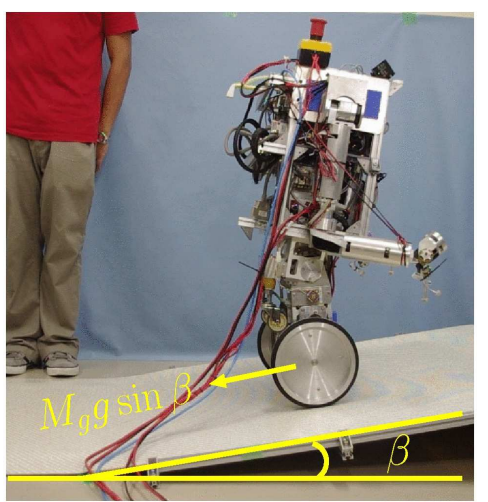

Fig. 9. Moving up a ramp

Traversing a ramp. The benefit of using the feedforward compensator can be seen when the robot traverses a ramp with a 10 degree slope (Fig. 9). Here, the mass of the robot becomes a disturbance opposing traversal and generating error in the wheel displacement. Figure 10 shows the reference wheel displacement and the measured wheel displacements of when the disturbance observer was applied and not applied. It is clear that the error in the wheel displacement is drastically reduced to $0.1 \mathrm{~m}$ when the compensator was used, while the error was approximately $0.4 \mathrm{~m}$ when not used.

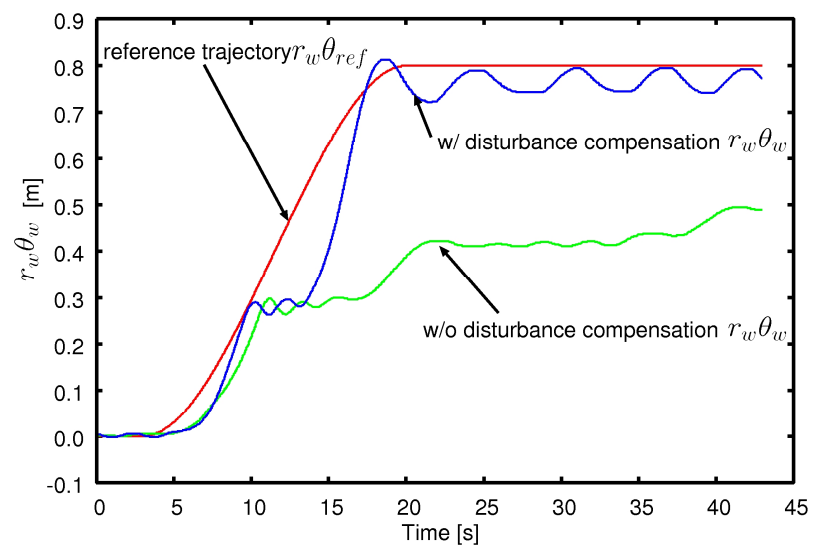

Fig. 10. Wheel Trajectory and wheel displacements for traversing a ramp

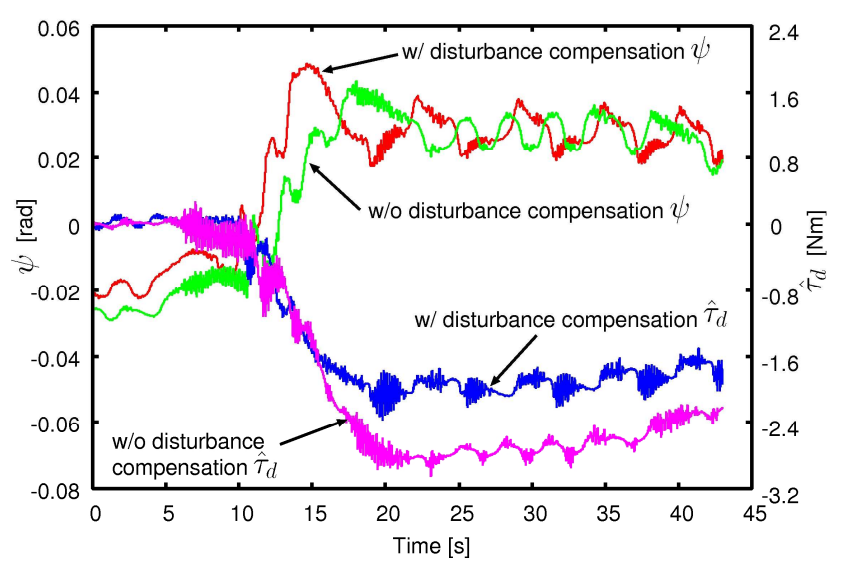

Fig. 11. Inclination Angle and estimated disturbance for traversing a ramp 


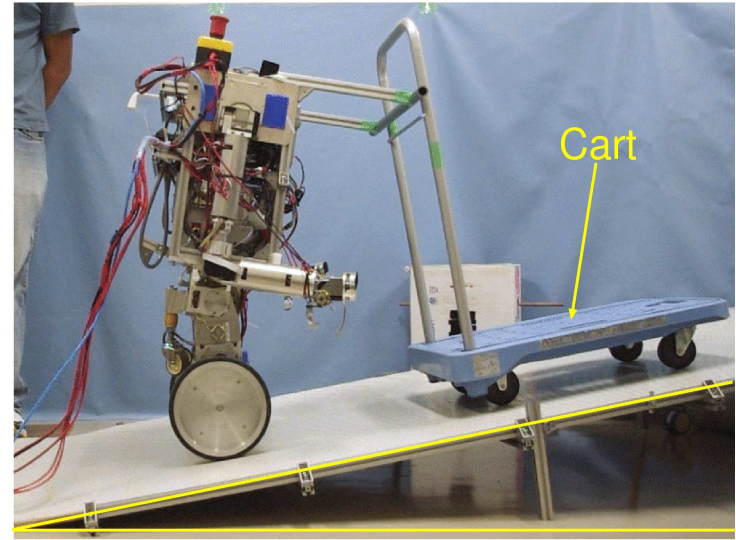

Fig. 12. Pushing a cart up a ramp motion

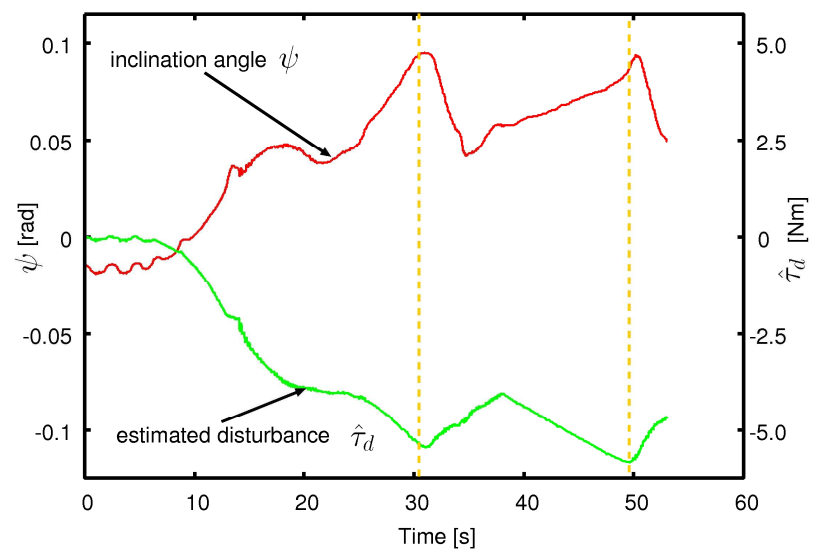

Fig. 14 Inclination angle and estimated disturbance for pushing a cart up a ramp

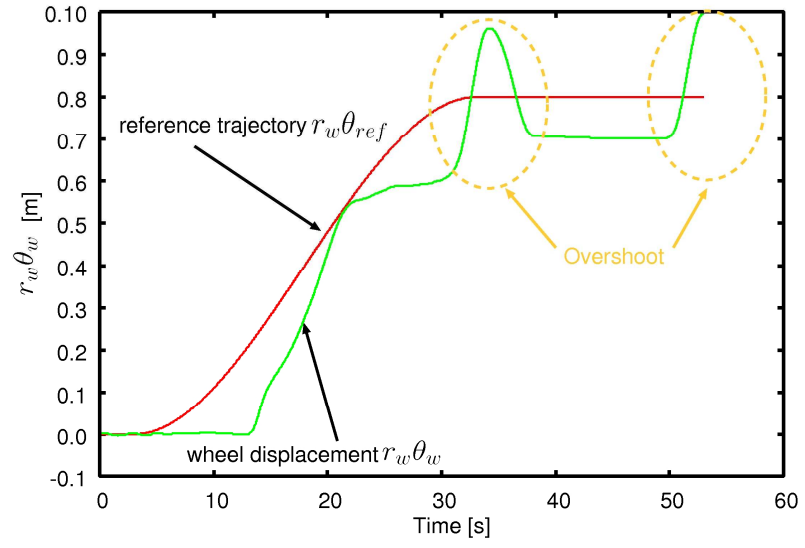

Fig. 13. Wheel trajectory and displacement for pushing a cart up a ramp

Pushing a cart up a ramp. Figure 12 shows the experiment performed for pushing a cart up a ramp and Fig. 13, shows the input wheel trajectory and the actual wheel trajectory of the robot. Comparison between the disturbance of traversing the ramp (Fig. 11) and pushing the cart up the ramp (Fig. 14) shows the difference in the kind of disturbance that occur. Pushing the cart up the ramp contains friction forces that have a higher values during low speeds and decrease once the minimum required force is overcome by the compensator and the cart begins to move. This can be seen in the peeking points in the inclination angle and estimated disturbance (marked by broken lines in Fig.14). The overshoot in the response occurs after these peeking points because the disturbance suddenly decreases and the speed of estimation is not fast enough. However, the error in the wheel displacement is reduced to $0.12 \mathrm{~m}$ in the steady state and so good disturbance regulation is achieved.

\section{Summary}

The reduced disturbance observer performed well in estimating the value of the disturbance. Its estimation time could be improved further to enable faster compensation of the actual disturbance value. The current implementation of the feedforward compensator is a simple static gain and although it reduced the error in traversing a ramp, compensation for frictional forces require a more dynamic compensator. Future work for the pushing and pulling action of the I-PENTAR will involve modeling and compensation of friction and the use of the arms for pushing and pulling.

\section{References}

[1] S. Kimura, S. Jeong, L. Canete, T. Takahashi, Load lifting motion for an unknown mass for a wheeled inverted pendulum, Proceedings of the 2011 JSME Conference on Robotics and Mechatronics, 1P1-I04.

[2] S. Kimura, S. Jeong, T. Takahashi, A state transition control for an inverted pendulum robot, Proceedings of the 28TH Annual Conferenceof the Robotics Society of Japan, 2Q2-8.

[3] J. Bay, Fundamentals of linear state space systems, 2nd ed., New York,McGraw-Hill, 1998 